\title{
Molecular Dynamics Simulations of Ibuprofen Release from pH- Gated Silica Nanochannels
}

\author{
Javier Rodriguez ${ }^{* \dagger, \dagger}$ and M. Dolores Elola ${ }^{\dagger}$ \\ ${ }^{\dagger}$ Departamento de Física, Comisión Nacional de Energía Atómica, Avenida Libertador 8250, 1429 Buenos Aires, Argentina \\ ${ }^{\ddagger}$ ECyT, UNSAM, Martín de Irigoyen 3100, 1650 San Martín, Provincia de Buenos Aires, Argentina
}

ABSTRACT: The iboprufen delivery process from cylindrical silica pores of 3 $\mathrm{nm}$ diameter, with polyamine chains anchored at the pore outlets, was investigated by means of massive molecular dynamics simulations. Effects from $\mathrm{pH}$ were introduced by considering polyamine chains with different degrees of protonation. High, low, and intermediate $\mathrm{pH}$ environments were investigated. The increment of the acidity of the environment leads to a significant decrease of the pore aperture, yielding an effective diameter, for the lowest $\mathrm{pH}$ case, that is

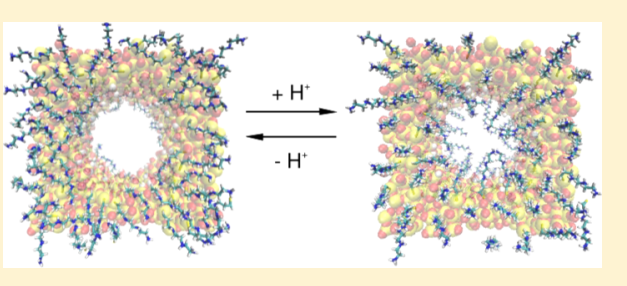
3.5 times smaller than the one associated with the highest $\mathrm{pH}$ one. Using a biased sampling procedure, Gibbs free energy profiles for the ibuprofen delivery process were obtained. The joint analysis of the corresponding profiles, time evolution of the ibuprofen position within the channel, orientation of the molecule, and instantaneous effective diameter of the gate suggest a three-step mechanism for ibuprofen delivery. A complementary analysis of the translational mobility of ibuprofen along the axial direction of the channel revealed a subdiffusive dynamics in the low and intermediate $\mathrm{pH}$ cases. Deviations from Brownian diffusive dynamics are discussed and compared with direct experimental results.

\section{INTRODUCTION}

Over the past two decades, impressive progress in selfassembled material science has been achieved. The cornerstone of this development stems from the design of structures through a stepwise synthesis of a wide variety of molecular architectures via covalent bonds, molecular stacking, dipoledipole association, and hydrogen bonds. ${ }^{1}$ Recently, the possibility of attaching functional molecular entities to preorganized nanostructures has been proposed as a means to explore new functional aspects of supramolecular assemblies. ${ }^{2-4}$ Important advances have been achieved, in areas such as self-assembled monolayers ${ }^{5,6}$ and nanoparticles, ${ }^{7-10}$ hostguest complexation on surfaces, ${ }^{1-15}$ and molecular machines, ${ }^{16-20}$ to cite a few relevant examples. On the other hand, the investigation of controlled drug delivery processes remains an issue of crucial interest in many fields, such as biology, chemistry, pharmacology, and medicine. ${ }^{21-25}$ In this particular context, mesoporous silica assemblies represent attractive building materials for drug reservoirs due to their unique properties, i.e., high surface-to-volume ratio, inertness, thermal stability, and homogeneous pore distribution. Moreover, the nanometer scale in pore sizes, that allows the encapsulation of a variety of solutes, along with the flexibility of the synthesis protocols and ease of functionalization permit, in turn, a tuning of the surface properties according to specific requirements. ${ }^{26-31}$ Still, a controlled drug release process requires the presence of a tunable gate capable of modulating material exchange with the environment.

Casasús and co-workers have recently designed a $\mathrm{pH}$-driven gate-like supramolecular system, by anchoring suitable polyamines at the pore outlets of mesoporous materials. ${ }^{32,33}$ At high
$\mathrm{pH}$ values, hydrogen-bond interactions between polyamine chains give rise to a poor coverage of the pore aperture, leading to a fully open gate; on the other hand, at low $\mathrm{pH}$ values, electrostatic repulsions between protonated polyamine species anchored at the pore rims promote a clear reduction-or even a closure-of the pore aperture. In a related context, Gao et al. ${ }^{34-36}$ have recently prepared and analyzed mesoporous silica spheres with switchable gates, by grafting multiamine chains around the mesopore outlets. In their ibuprofen (IBU) release test, they obtained a high response to the $\mathrm{pH}$ : IBU was released rapidly and completely at high $\mathrm{pH}$ 's, whereas, at low $\mathrm{pH}$ 's, only a small fraction of ibuprofen (15 wt \%) was delivered from the nanopores.

Despite the apparent simplicity of the interactions involved in the open/close process, many aspects related to the drug release mechanisms and the characteristics of the host structure remain poorly understood. Consequently, our interest here focused on the elucidation of the microscopic details that regulate the mentioned process, within the microscopic framework provided by computer simulations.

In the present paper, we will present results from molecular dynamics (MD) simulations performed on a model mesoporous system, containing a single IBU solute inmersed in liquid water and trapped within a cylindrical silica nanochannel of diameter $\sim 3 \mathrm{~nm}$, whose lateral outlets are decorated with polyamine chains. The particular choice of ibuprofen as the

Special Issue: Branka M. Ladanyi Festschrift

Received: June 5, 2014

Revised: July 30, 2014 
trapped solute is based on its moderate size, its relatively simple molecular geometry, and its major relevance as a typical test molecule in controlled drug delivery experiments. Effects from the $\mathrm{pH}$ associated with the liquid solution were introduced in the simulations by considering polyamine chains with different degrees of protonation.

Previous theoretical studies have been performed on similar silica/polyamine nanochannels, by the same groups that reported direct experimental measurements. ${ }^{4,33}$ However, it is worth mentioning some relevant differences between these previous simulations and the present study. Basically, our model includes a much more detailed description of the molecular architecture of the pore, much longer trajectories at temperatures closer to the experimental conditions, and a more realistic characterization of the aqueous solvent by the explicit incorporation of water molecules.

The organization of the paper is as follows: In section 2, we summarize the details of the model and the technical aspects of the simulation procedure. Section 3 contains the main results of our work, in which we examine and discuss the structural features of the host nanocomposite and the ibuprofen delivery process. The conclusions are left for section 4 .

\section{MODEL AND SIMULATION DETAILS}

The systems investigated were composed of a single IBU solute embedded within a water-saturated, cylindrical silica pore of $\sim 3$ $\mathrm{nm}$ diameter, with polyamine chains attached at the pore outlets (see scheme in Figure 1). All different components were modeled as a collection of interacting sites. As usual, the overall potential energy of the system was decomposed into a sum of site-site, pairwise interactions combining dispersion (LennardJones) and Coulomb contributions.

The SPC/E potential was adopted for water interactions. ${ }^{37}$ The inter- and intramolecular potential parameters employed for silica and silanol groups were taken from ref 38. The polyamine molecules were modeled as fully flexible units, including a set of 29-32 interacting sites, depending on the degree of protonation. Intra- and intermolecular interactions involving these sites and the counterions $\mathrm{Cl}^{-}$were taken from the CHARMM27 force field. ${ }^{39}$ The united-atom CHARMM19 force field was used to model ibuprofen interactions. ${ }^{40}$ This model for ibuprofen has already been employed in previous works; a detailed description of its applicability and testing can be found elsewhere. ${ }^{41,42}$

The preparation of the system involved a sequential procedure: First, a cylindrical silica pore of radius $16 \AA$ was built up from an amorphous silica block of dimensions $L_{x}=L_{y}$ $=50 \AA, L_{z}=30 \AA$. . Details of this procedure can be found in previous works. ${ }^{43,44}$ Silanol groups, adsorbed at the internal pore walls, were included, with a surface density of $\rho_{\mathrm{SiOH}} \sim 3$ $\mathrm{nm}^{-2}$, in agreement with experimental absorption data. ${ }^{45}$ Second, multiamine chains were located at the two lateral, external walls of the silica block, anchored at silicon sites with coordination numbers lower than 4 . The resulting surface density of multiamine was of the order of $\sim 3.5 \mathrm{~nm}^{-2}$, in agreement with experimental data. ${ }^{4,32}$ This procedure leads to the formation of a near monolayer of multiamines at the external silica walls, with a substantial number of polyamines around the outlet pore rims. A minor percentage of the polyamines was anchored at the internal surface of the silica nanochannel, close to the pore aperture, yielding an effective functionalization of the nanochannel of $\sim 1 \mathrm{~nm}$ deep, in order to mimic experimental conditions. ${ }^{4}$ The final stage involved the
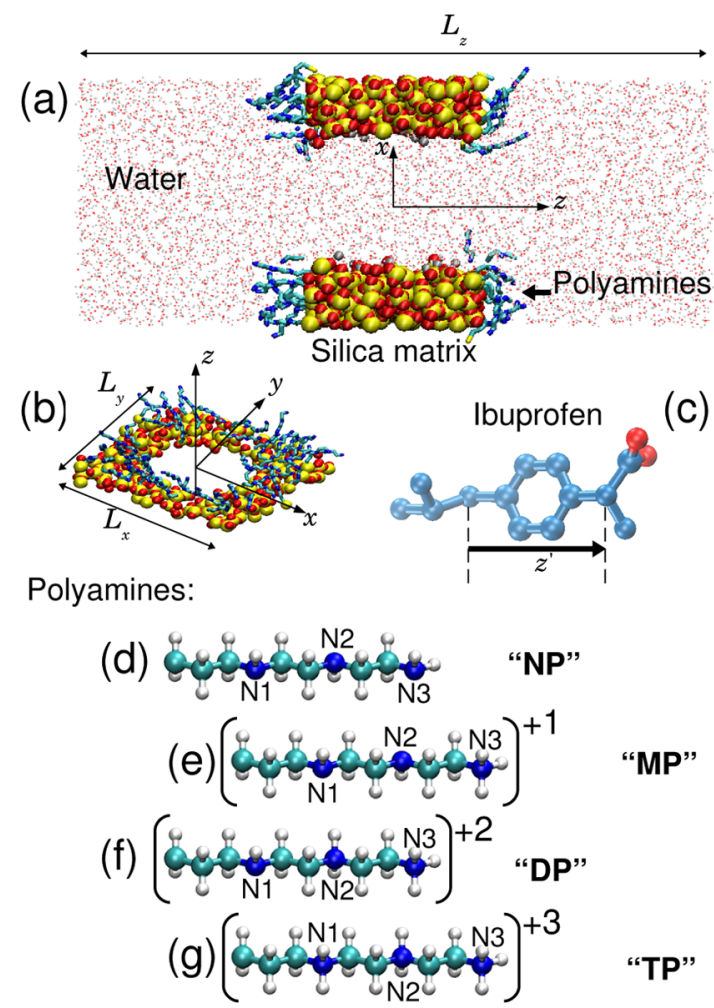

Figure 1. Representation of the simulated systems and detail of the polyamines considered. (a) axial cut of the system; (b) detail of the pore aperture; (c) ibuprofen molecule, as represented in the unitedatom model, with the $z^{\prime}$ axis fixed to the molecular frame, pointing toward the hydrophilic carboxylate group. The schemes depicted in panels $\mathrm{d}-\mathrm{g}$ correspond to the different polyamine substituents anchored at the silica pore outlets: (d) nonprotonated (NP), (e) monoprotonated (MP), (f) diprotonated (DP), and (g) triprotonated (TP) polyamine species.

incorporation of the water liquid phase into the system. To do so, the interior of the pore was filled with water, and two adjacent bulk-like water reservoirs of dimensions $50 \times 50 \times 65$ $\AA^{3}$ were brought in contact with the silica block at the pore rims. The overlapping water molecules were discarded, yielding a total number of waters of $N_{w} \sim 7300$. From then on, the liquid phase was allowed to reequilibrate at $T=300 \mathrm{~K}$ for about 4 ns. During this period, the length of the simulation box along the $z$-axis was adjusted to set the local density at the center of the water reservoir to the bulk value under ambient conditions, $\rho_{\text {bulk }}=0.033 \AA^{-3}$. The final size of the box length along the $z$ axis resulted in $L_{z} \approx 120 \AA$ (see Figure 1a).

It is well documented that multiamine chains are highly sensitive to the solution $\mathrm{pH} .{ }^{32}$ At high $\mathrm{pH}$ 's, multiamine chains remain close to each other via hydrogen bond interactions, whereas, at low $\mathrm{pH}$ 's, these moieties are most likely fully protonated and tend to separate from each other, due to electrostatic repulsions. In order to mimic the effects of the $\mathrm{pH}$ of the solution on the degree of protonation of the material, multiamines with different net charges were included in the model.

The $\mathrm{p} K_{\mathrm{a}}$ constants of a typical triamine in bulk water are of the order of 8.5, 9.6, and 11 (ref 46). However, since an acidic molecule anchored to a positively charged surface raises its acidic strength, ${ }^{47}$ one could predict that the $\mathrm{p} K_{\mathrm{a}}$ values will be substantially modified for the systems studied here. Titration measurements of polyamine/silica solid samples show that, 
although the stepwise $\mathrm{p} K_{\mathrm{a}}$ constants in water are shifted toward smaller values, the difference between subsequent $\mathrm{p} K_{\mathrm{a}}$ values is maintained within $\sim 1-1.5$ units. ${ }^{33}$

Nonprotonated neutral chains (NP) and protonated triamines with a net charge of $+1 e$ (monoprotonated, MP), $+2 e$ (diprotonated, $\mathrm{DP}$ ), and $+3 e$ (triprotonated, TP) were considered; see Figure $1 \mathrm{~d}-\mathrm{g}$. We assumed that, as the $\mathrm{pH}$ decreases, the protonation process occurs in a gradual fashion, starting by the most external terminal amine group of the chain $(\mathrm{N} 3)$, then at the intermediate one (N2), and finally at the amine group closest to the silicon anchoring site $(\mathrm{N} 1) .{ }^{48}$

We investigated three different silica/multiamine systems: (i) In the first one, the triamine monolayer was composed exclusively of nonprotonated NP species, mimicking a high$\mathrm{pH}$ environment; from now on, we will refer to this system as the unprotonated system (UPS). (ii) The second system corresponded to a low-pH solution, in which all the polyamines anchored at the pore outlets were triprotonated (TP) species. This system will be referred to as the fully protonated system (FPS). In this case, in order to maintain the charge neutrality of the system, we included adequate amounts of chloride counterions within the aqueous reservoirs. (iii) An intermediate system was also modeled, in which $45 \%$ of the polyamines were monoprotonated, $45 \%$ were diprotonated, $5 \%$ were nonprotonated, and 5\% were triprotonated. This combination yields an average charge per polyamine of $\left\langle q_{\text {amine }}\right\rangle=+1.5 e$. Appropriate amounts of chloride counterions were also included to keep charge neutrality.

In Table 1, we display the numbers of molecules of species of each simulated system. All molecular dynamics (MD)

\section{Table 1. Details of the Simulations}

\begin{tabular}{lrrrrrrl} 
system & $N_{\mathrm{NP}}$ & $N_{\mathrm{MP}}$ & $N_{\mathrm{DP}}$ & $N_{\mathrm{TP}}$ & $N_{\mathrm{Cl}^{-}}$ & $N_{\mathrm{w}}$ & $\left\langle q_{\text {amine }}\right\rangle$ \\
UPS & 104 & 0 & 0 & 0 & 0 & 7615 & 0 \\
HPS & 5 & 47 & 47 & 5 & 156 & 7319 & $+1.5 e$ \\
FPS & 0 & 0 & 0 & 104 & 312 & 7319 & $+3.0 e$ \\
\hline
\end{tabular}

trajectories were generated using the NAMD package ${ }^{49}$ and corresponded to microcanonical runs. Periodic boundary conditions were applied along the three Cartesian coordinates. Short-ranged intermolecular forces were cut off at $13 \AA$, while the particle mesh Ewald (PME) method was implemented to handle long-range Coulomb forces. The equations of motion were integrated using a multiple time step integration scheme, with a time step of 1 fs for intramolecular modes and nonbonded short-ranged forces, and $2 \mathrm{fs}$ for the rest of the Coulomb forces.
The final stage of the preparation of the systems involved the incorporation of IBU inside the silica pore. A single IBU molecule was inserted within the pore with its center of mass located initially at the center of the pore; overlapping water molecules were discarded. Soft harmonic interactions, with restoring forces of $k \sim 15 \mathrm{kcal} \mathrm{mol}^{-1} \AA^{-2}$, acting on the IBU center of mass and centered at the corresponding initial position, were applied on the $x$ and $y$ directions. This external potential helped to maintain the translational dynamics of IBU mostly along the $z$-axis.

\section{RESULTS}

3.1. Structural Features of the Host Nanocomposite. We will start our analysis by investigating the structural characteristics of the silica/polyamine host nanocomposite. To that aim, we will first explore the local density fields associated with the polyamine chains along directions perpendicular to the pore axis, in order to estimate the effective diameter of the pore opening. In Figure 2, we present results for two-dimensional maps, $\rho_{\text {amine }}(x, y)$, of the type

$$
\rho_{\text {amine }}(x, y) \propto \sum_{N_{\text {amine }}} \sum_{i}\left(\left\langle\delta\left(x_{i}-x\right)\right\rangle,\left\langle\delta\left(y_{i}-y\right)\right\rangle\right)
$$

where $x_{i}$ and $y_{i}$ are the coordinates of the $i$ th site of polyamine molecules projected onto the basal plane $z=0$, and considering all interacting sites of the polyamine molecules. Figure 2 also shows, as a reference, the contour of the silica pore. At first glance, the open/closed states, corresponding to the systems containing NP and TP polyamines, respectively, are clearly visible. The NP multiamines display poor coverage of the pore, resulting in an "open gate". In contrast, the FPS system exhibits a substantial reduction of the pore aperture, associated with a "closed gate". The plots also reveal an increasing degree of rigidity of the polyamine chains with their protonation. Since all density maps have been averaged over MD trajectories of similar length (15 ns), the loss of homogeneity exhibited in the $\rho_{\text {amine }}(x, y)$ maps suggests a reduction of the mobility of the multiamine chains.

The effective size of the pore aperture was quantitatively estimated from the effective pore areas, computed from a particle insertion method. ${ }^{50}$ Briefly, the polyamine sites at each pore rim were projected onto a basal plane and test particles were randomly inserted in this plane. Only those test particles which showed no polyamine neighbors within a $3 \AA$ distance were "accepted". After $M=20000$ insertion trials, the effective area $A_{\text {eff }}$ of the pore aperture was calculated according to (a)

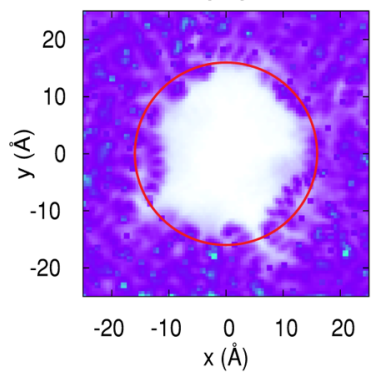

(b)

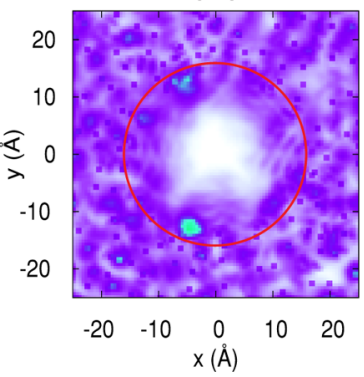

(c)

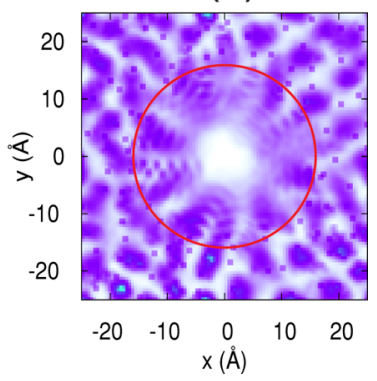

Figure 2. Local density maps of multiamine chain species, projected on the $x y$ plane. The circular lines represent the diameter of the silica pore. Panels a, b, and c correspond to the systems UPS, HPS, and FPS, respectively. 


$$
A_{\text {eff }}=A_{\mathrm{p}} \frac{M_{\mathrm{acc}}}{M}
$$

where $A_{\mathrm{p}}$ is the area over which the test particles are randomly shot and $M_{\text {acc }}$ is the number of accepted test particles. This trial insertion process of test particles was repeated for each stored configuration, and an average value for $A_{\text {eff }}$ was finally obtained. Assuming a circular geometry, the effective diameter of the pores can be estimated as $d=2\left(A_{\text {eff }} / \pi\right)^{1 / 2}$. The effective sizes were found to be $d=23.9,12.1$, and $6.8 \AA$ for UPS, HPS, and FPS systems, respectively, and are shown in Figure 3 as a function of $\left\langle q_{\text {amine }}\right\rangle$, the average charge per polyamine molecule.

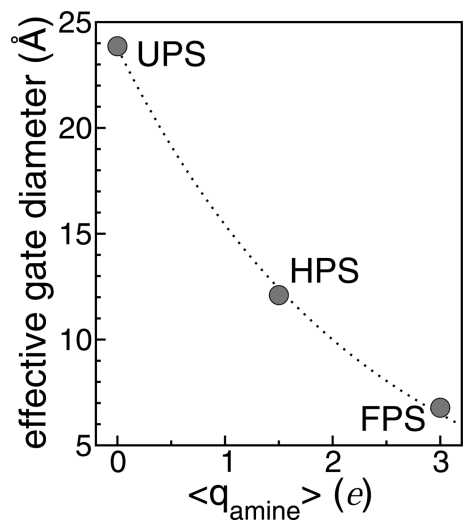

Figure 3. Effective radius as a function of the average charge per multiamine molecule corresponding to each of the simulated systems.

Density profiles of some relevant species have also been calculated, in cylindrical coordinates, according to

$$
\rho_{\alpha}\left(r^{\prime}\right)=\frac{1}{2 \pi r^{\prime} L_{z}} \sum_{i}\left\langle\delta\left(R_{i}^{\alpha}-r^{\prime}\right)\right\rangle
$$

where $R_{i}^{\alpha}=\left[\left(x_{i}^{\alpha}\right)^{2}+\left(y_{i}^{\alpha}\right)^{2}\right]^{1 / 2}$ denotes the distance between the position of site $i$ in the molecule of species $\alpha$ and the pore axis. Figure 4 shows the radial profiles of silanol, water, and

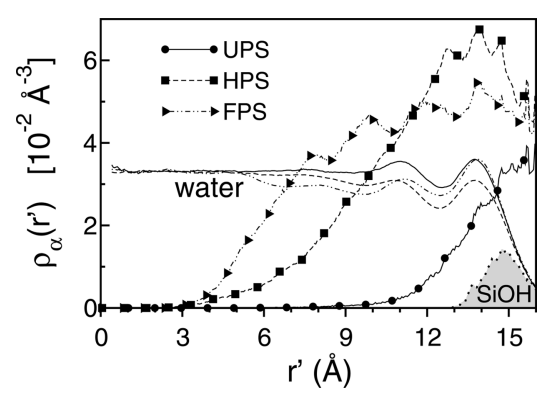

Figure 4. Local density profiles along the cylindrical radial direction, for the different species in the systems studied. Solid, dashed, and dotdashed lines correspond to water density, respectively; the curves with symbols, to the polyamine species, as indicated.

polyamine species. Water profiles exhibit little variations: In all cases, a two-layer aqueous structure is observed near the silica walls, in the range $r \approx 10-16 \AA$, whereas, for more internal locations, i.e., $r \lesssim 10 \AA$, the water bulk density, $\rho_{\text {bulk }}=0.033$ $\AA^{-3}$, is attained. The most outer aqueous layer corresponds to water molecules involved in hydrogen bonding ( $\mathrm{HB}$ ) with the silanol groups adsorbed at the interface (see silanol shaded peak at $r \approx 15 \AA$ ). The reduction in the pore aperture appears clearly in Figure 4, where the threshold radius at which the multiamine density starts to exhibit non-negligible values is $r \sim 3.5 \AA$ for FPS ( $r \sim 11 \AA$ for the UPS system). These numbers, in turn, agree with estimations of the pore apertures based on results from the particle insertion method.

In order to analyze the variations in the solvation structures around the multiamine species, we computed site-site pair correlation functions between pairs of atoms involving the most external nitrogen site (N3) of polyamine species, namely,

$$
g_{\alpha-\beta}=\frac{1}{4 \pi r^{2} N_{\alpha}} \sum_{i}^{N_{\alpha}} \sum_{j \neq i}^{N_{\beta}}\left\langle\delta\left(r-r_{i j}^{\alpha \beta}\right)\right\rangle
$$

where $N_{\alpha}$ represents the number of sites of species $\alpha$ and $r_{i j}^{\alpha \beta}=\left|\mathbf{r}_{i}^{\alpha}-\mathbf{r}_{j}^{\beta}\right|$. Of course, due to the lack of spherical symmetry in these systems, a gradual decrease of the asymptotic long- $r$ behavior is expected. The top panel of Figure 5 shows pair

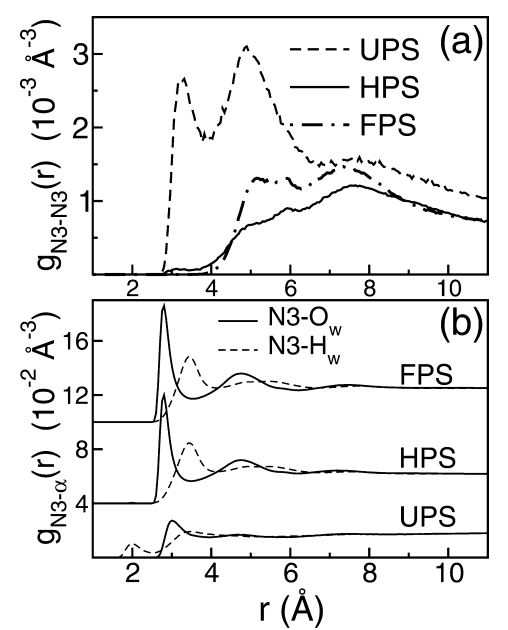

Figure 5. Site-site radial pair correlation function for (a) N3-N3 pairs of polyamine species and (b) N3-water pairs, for the systems studied. The curves associated with HPS and FPS systems in panel $b$ have been shifted vertically by +4 and $+10 \times 10^{-2} \AA^{-3}$, respectively.

correlations between N3 sites, $g_{\mathrm{N} 3-\mathrm{N} 3}(r)$. The curves displayed in Figure 5a reveal a gradual increment of the electrostatic repulsive interactions between polyamine species with the degree of protonation. For the UPS system, the first maximum is found at the shortest separations, $r=3.2 \AA$, and can be associated mainly with direct HB between NP chains; we shall refer to these HBs as "HB1". The second peak observed in $g_{\mathrm{N} 3-\mathrm{N} 3}$ for the UPS system, at $r \approx 5 \AA$, higher in magnitude than the first maximum, corresponds to the second "solvation shell" of the tagged N3 site, which contains $\sim 2.2 \mathrm{~N} 3$ neighbors. For the intermediate HPS system, the maximum of $g_{\mathrm{N} 3-\mathrm{N} 3}$ at $r=3.2$ $\AA$ practically vanishes, due to the protonation of an important fraction of these terminal sites, and to the gradual loss of the $\mathrm{HB}$ acceptor character of the $\mathrm{N} 3$ atoms. As a result, the number of HB1 bonds diminishes substantially.

On the other hand, in order to shed light on the role of water molecules in the open/close mechanism, a complementary analysis of pair correlations between $\mathrm{N} 3$ and water sites $\left(\mathrm{O}_{\mathrm{w}}\right.$, $\mathrm{H}_{\mathrm{w}}$ ) was performed. Those pair correlations are depicted in Figure $5 \mathrm{~b}$. As one can see, in UPS, $g_{\mathrm{N} 3-\mathrm{Ow}}(r)$ has a first maximum at $r \approx 3 \AA$, whereas that of $g_{\mathrm{N} 3-\mathrm{Hw}}(r)$ lies at shorter separations, $r=2 \AA$, suggesting a HB connectivity of the type $\mathrm{N} 3 \cdots \mathrm{H}_{\mathrm{w}}-\mathrm{O}_{\mathrm{w}}$ (multiamine/water-donor, hereafter referred to as 
"HB2"). One can conclude that polyamines in UPS are primarly connected through direct $\mathrm{HB}$ between $\mathrm{N} 3$ terminal sites (HB1), and also through bridging water molecules (HB2), as represented schematically in Figure 6. Regarding water

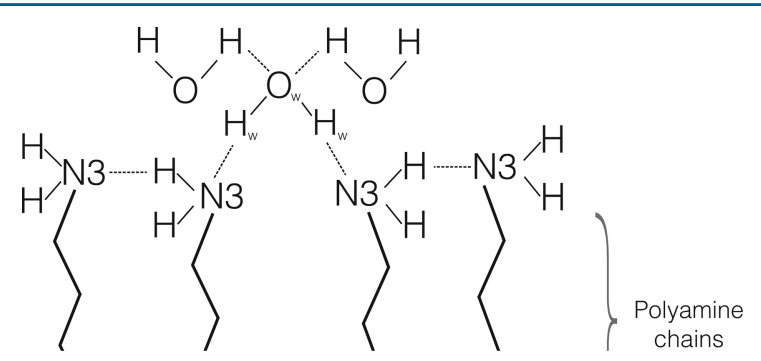

Figure 6. Pictorial representation of a possible solvation structure around polyamine terminal sites N3, for the UPS system (see text).

solvation around N3 sites in HPS and FPS systems, note that the first peak of $g_{\mathrm{N} 3-\mathrm{Ow}}(r)$ is higher and shifted toward shorter distances than in the UPS one, whereas that of $g_{\mathrm{N} 3-\mathrm{Hw}}$ at $r=2$ $\AA$ disappeared. Consequently, we conclude that HB2 bonds practically disappear as the acidity of the environment increases, as well as the direct $\mathrm{HB} 1$ connections, in favor of stronger HBs of the type multiamine/water-acceptor. The higher magnitude of $g_{\mathrm{N} 3-\mathrm{N} 3}$ in the FPS than in the HPS might be interpreted in terms of $\mathrm{N} 3$ terminal sites of polyamine that become gradually more favorably solvated by water and chloride as the acidity of the solution increases. Specifically, the N3-N3 coordination is bridged by water and chloride molecules around N3, a configuration that in the FPS appears as more compact than in the intermediate HPS case.

Figure 7 shows local density profiles of some relevant species along the axial axis, computed as

$$
\rho_{\alpha}(z)=\frac{1}{A} \sum_{i}\left\langle\delta\left(z_{i}^{\alpha}-z\right)\right\rangle
$$

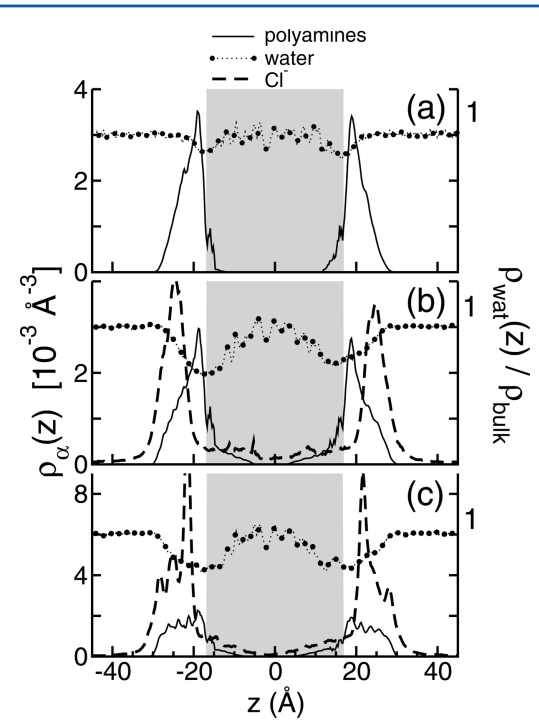

Figure 7. Local density profiles along the axial direction $z$, for the polyamine sites (solid lines) and chloride anions (dashed lines), in the different systems: (a) UPS, (b) HPS, and (c) FPS. The water density (right axis) was computed within a cylinder of $15 \AA$ radius and $120 \AA$ length, and normalized with $\rho_{\text {bulk }}=0.033 \AA^{-3}$, the aqueous bulk density. The shaded region indicates the location of the silica block. where $z_{i}^{\alpha}$ is the $z$-coordinate of the $i$ th site in the molecule of species $\alpha$. The profiles displayed in Figure 7 show that, inside the pore and beyond $|z| \gtrsim 30 \AA$, the water density reached the bulk value. At the pore rims, however, the water density falls down to $70 \%$ of the latter value. The profiles also show that the chloride counteranions locate most likely in the same region where polyamine chains are found. The profiles associated with the FPS system reveal that the $\mathrm{Cl}^{-}$anions are arranged in layers along the $z$-axis, with their maxima overlapping those of the protonated groups of the polyamine chains. Figure 8 displays

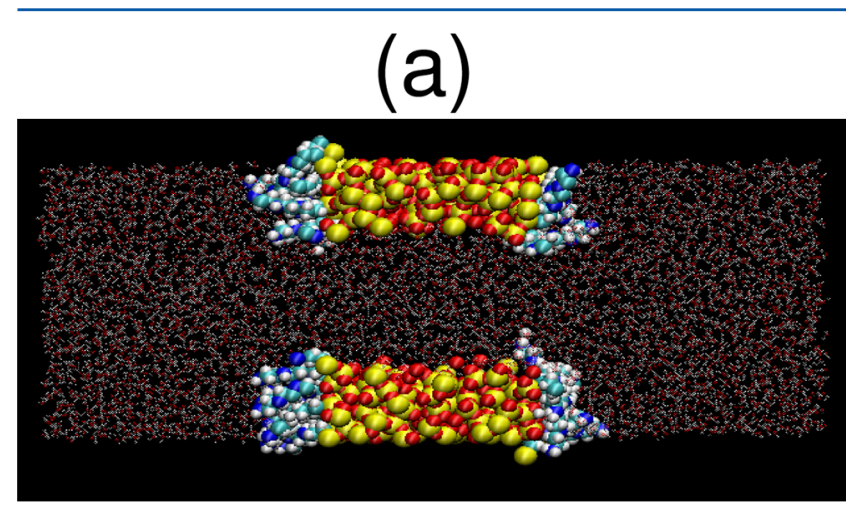

(b)

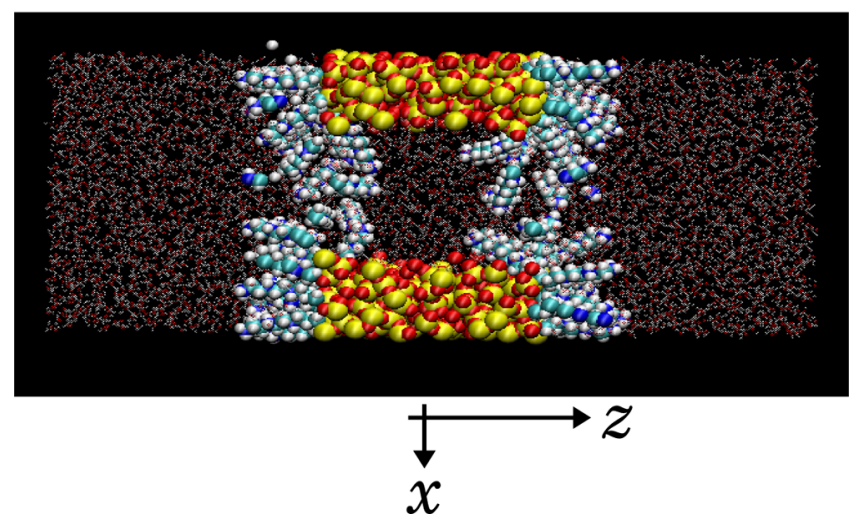

Figure 8. Axial cut of two snapshots captured during the MD simulations for (a) the UPS system and (b) the FPS one. Silanol and chloride species are not shown for clarity.

axial cuts of two typical snapshots of the UPS and FPS systems during the MD simulations, where different orientations of the polyamine chains at the pore outlets can be observed.

The computation of the average length of the polyamine chains, $l=\left\langle\left|\mathbf{r}_{i}^{\mathrm{N} 3}-\mathbf{r}_{i}^{\mathrm{S} \mathrm{i}}\right|\right\rangle$, shows small fluctuations, ranging from $l$ $=11.4 \AA$ for the UPS to slightly more stretched values of $l=$ $12.7 \AA$ for the FPS system. Much more marked changes were observed in the orientation of the polyamine chains with respect to the axial $z$-axis, $\cos \theta=\left\langle\mathbf{r}_{i}^{\mathrm{Si}-\mathrm{N} 3} \cdot \hat{z} /\left|\mathbf{r}_{i}^{\mathrm{Si}-\mathrm{N} 3}\right|\right\rangle$, shown in Figure 9, where panels a and $\mathrm{b}$ correspond to the polyamine chains anchored at silicon sites located at $r \leq 20 \AA$ (near the pore rims) and $r>20 \AA$ (away from the pore borders).

The probability distributions displayed in Figure 9 indicate that (i) for the UPS system, the main maximum of polyamine chain orientations corresponds to an angle of $\sim 40^{\circ}$; (ii) the orientational correlations associated with polyamine chains located near the pore $(r \leq 20 \AA)$ exhibit a bimodal character at low and intermediate $\mathrm{pH}$ 's, corresponding to $\theta \approx 120^{\circ}$ (i.e., polyamine species pointing toward the interior of the pore 

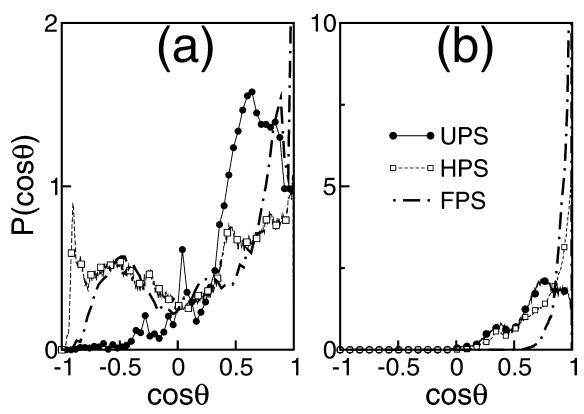

Figure 9. Orientational correlation, $P(\cos \theta)$, for the polyamine chains anchored at distances (a) smaller than $20 \AA$ with respect to the axis of the pore and (b) greater than $20 \AA$ to the pore axis.

cavity), and a second peak near $\cos \theta \sim 1$; (iii) the larger intensity and narrower shape of the maximum at $\cos \theta \sim 1$ for FPS (panel b) reveal that the most external polyamine chains exhibit a more marked parallel orientation with the degree of protonation.

3.2. Ibuprofen Delivery and Free Energy Profiles. We will now investigate the mechanisms of drug delivery through the polyamine gates in solutions at different $\mathrm{pH}$ values. The simplest geometrical order parameter, $\xi$, that describes the degree of release of IBU through the silica/polyamine outlets is

$$
\xi=Z_{\mathrm{IBU}}-Z^{0}
$$

In the previous equation, $Z_{\mathrm{IBU}}$ and $Z^{0}$ stand for the $z$ coordinates of the centers of mass of the IBU molecule and of the water sample, respectively. In this context, the magnitude of interest is given by the Gibbs free energy associated with the order parameter

$$
-\beta G(\tilde{\xi}) \propto \ln \langle\delta(\xi-\tilde{\xi})\rangle
$$

Sampling of all relevant fluctuations along different values of $\xi$ was performed by implementing an adaptive biasing force (ABF) scheme. ${ }^{51,52}$ This methodology has been successfully employed in studies of cyclodextrin encapsulation processes involving simple guest molecules, such as the perchlorate ion, ${ }^{53}$ or even more complex guests, such as cholesterol ${ }^{54}$ or rotaxane molecules. ${ }^{55}$ The ABF scheme relies on the generation of trajectories along a chosen reaction coordinate, experiencing practically no free energy barriers. This is achieved by means of biasing forces estimated along a series of small bins which, in turn, span the complete $\xi$ interval. These forces are applied to flatten the free energy surface, so that $\xi$ becomes uniformly sampled. The $\mathrm{ABF}$ calculations involved the use of 15 evenly distributed windows of width $4 \AA$, to fully cover the range of the reaction coordinate during the solute delivery. Within each window, the average force was accumulated in 0.1 A sized bins. The application of the adaptive bias was initiated after the accumulation of 1000 samples in individual bins, to avoid nonequilibrium effects. Production runs in each window lasted for $1.5 \mathrm{~ns}$. A uniform sampling, of $5 \times 10^{4}$ samples per bin, was obtained using those $\mathrm{ABF}$ parameters, meaning that the free energy profiles are well converged.

The free energy profiles for the release of IBU through the silica/polyamine pore outlets are shown in Figure 10. As a reference, the plot includes two shaded regions, indicating the location of the silica block itself (light gray) and the region occupied mainly by the polyamine chains (dark gray). For the IBU in the UPS, the profile of $\Delta G$ exhibits a small barrier at the center of the pore, followed by a shallow well in the range $5 \AA$

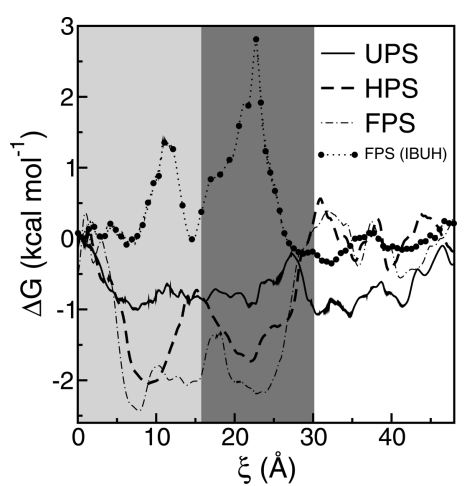

Figure 10. Gibbs free energy profiles for the release of IBU from a silica pore with polyamine outlet decorations. Light and dark shaded regions indicate the location of the silica block and the polyamine chains, respectively. The line with symbols corresponds to the release profile of the protonated form of ibuprofen, IBUH, in the FPS system (see text).

$\leq \xi \leq 45 \AA$, of depth $\sim 1 \mathrm{kcal} \mathrm{mol}^{-1}$. An $\sim 0.8 \mathrm{kcal} \mathrm{mol}^{-1}$ barrier at $\xi \sim 27 \AA$ is also observed. At this point, it is important to stress that the release of the IBU through the pore outlets corresponds to a passage of the probe from low to high solvent density regions. Consequently, a careful analysis of the microscopic features that characterize this process reveals the following: (i) There is a net loss of $\sim 3$ water-water HBs, associated with the annihilation of the solute cavity within the internal-less dense-region and the accommodation of the probe within the external bulk phase. (ii) A net gain of $\sim 1$ IBU-water $\mathrm{HB}$ is found, mainly associated with a stronger interaction between IBU and the external solvent. We tend to believe that the latter barrier, at $\xi \sim 27 \AA$, is originated in the subtle interplay of water-water and IBU-water contributions to $\Delta G$, that mainly vary in a narrow spatial range near the channel gate.

The free energy profile of IBU in the HPS system exhibits an additional $\sim 1 \mathrm{kcal} \mathrm{mol}^{-1}$ barrier, centered at the pore rim, $\xi \sim$ $15 \AA$, flanked by two nearly symmetric local minima: one located on the silica pore side and a second one at $\xi \sim 23 \AA$, a region where the polyamine chains and chloride species prevail. For the FPS case, the energy profile looks similar in shape to the latter, although the height of the barrier is slightly smaller; on the other hand, the depths of the two nearly symmetric wells around the barrier are somewhat larger than those for the HPS system. The profiles indicate that the global energetic costs for the IBU release are both of the order of $2.5 \mathrm{kcal} \mathrm{mol}^{-1}$ in FPS and HPS systems.

In order to gain a microscopic insight about the mechanisms of the ibuprofen release through the silica/polyamine nanocomposite, in the protonated HPS and FPS cases, we monitored the time evolution of a few relevant parameters along a $30 \mathrm{~ns}$, equilibrium trajectory, with the IBU solute initially located inside the pore, at $\xi \approx 0$. In the top panels of Figures 11 and 12, we have displayed the time evolution of the order parameter, $\xi(t)$. The shaded region indicates the limits of the solid silica block. Note that in the FPS and HPS systems the IBU release occurs at $t \approx 17$ ns. A few "frustrated" events at earlier times are perceived in the latter case, in which the IBU was able to reach the pore gate region (at $t \approx 1.5,5.5,10$, and $13 \mathrm{~ns}$ ) but the release was hindered by the environment. 


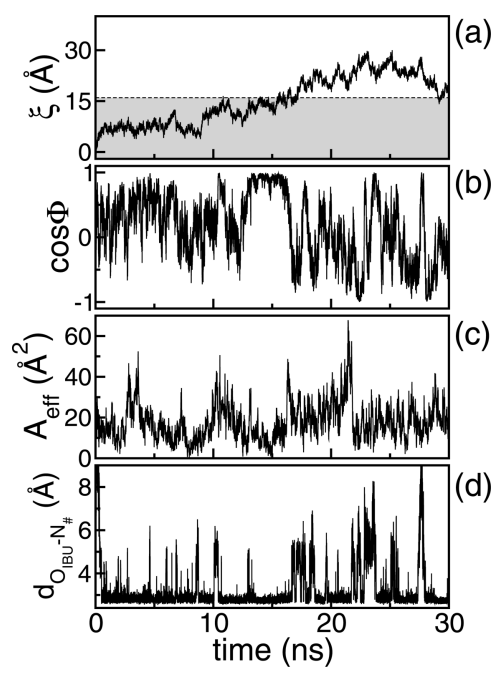

Figure 11. Time evolution of selected parameters during the MD trajectory of IBU release. (FPS System)

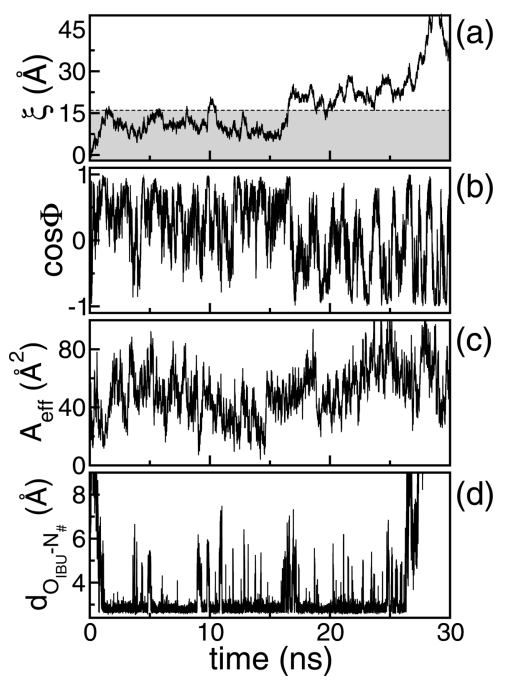

Figure 12. Time evolution of selected parameters during the MD trajectory of IBU release. (HPS System)

The orientation of IBU solute with respect to the axial axis can be monitored by analyzing the following orientational variable

$$
\cos \Phi=\hat{z}^{\prime} \cdot(\xi \hat{z}) /|\xi|
$$

where $\hat{z}^{\prime}$ is a unit vector along the IBU axis shown in Figure 1c.

The simultaneous analysis of $\xi(t)$ and $\cos \Phi(t)$ shows that, immediately after the release of the solute from the pore cavity, a sudden change in its orientation takes place, as $\cos \Phi$ changes from values around unity down to negative ones (see profiles in panel $\mathrm{b}$ of Figures 11 and 12 at $t \approx 17 \mathrm{~ns}$ ). A closer inspection of some snapshots captured from the MD trajectory suggests that IBU delivery includes a first stage in which the hydrophilic carboxylate group stacks at the pore gate, pointing toward the bulk solution; a second step follows, that involves a rigid rotation around the stacking point. As a result, in the final stage, the hydrophobic moiety of IBU points toward the outer bulk solution, whereas the carboxylate one, toward the interior of the silica pore. Three representative snapshots that illustrate the above observations are displayed in Figure 13. The configurations shown in Figure 13a and c could be ascribed to the two folded minima observed in the energy profiles of HPS and FPS systems; meanwhile, snapshot $b$ of Figure 13 represents a configuration corresponding to $\xi \approx 15 \AA$, the position of the intermediate energy maximum of $\Delta G$.

The time evolution of the effective area of the pore aperture, shown in panel $\mathrm{c}$ of Figures 11 and 12, reveals that, although important fluctuations are present in $A_{\text {eff }}(t)$ along the whole trajectory, the IBU release occurs concomitantly with a fast opening of the gate, followed by a sudden drop in $A_{\text {eff. }}$ The bottom panels of Figures 11 and 12 provide complementary observables that are consistent with the three-step IBU delivery mechanism described above. The curves depict the distance between oxygen sites of IBU and the closest nitrogen site of any

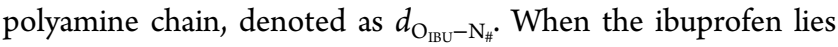
away from the decorated pore rims, $d_{\mathrm{O}_{\mathrm{IBU}}-\mathrm{N}_{\#}}$ takes values above $\sim 8 \AA$, whereas, as IBU moves closer to the pore aperture, the distance $d_{\mathrm{O}_{\mathrm{IBU}}-\mathrm{N}_{\#}}$ may go down to $3 \AA$. Note that the solute delivery from FPS and HPS systems occurs after a short period during which $d_{\mathrm{O}_{\mathrm{IBU}}-\mathrm{N}_{\#}}$ remains close to $3 \AA$, revealing that the anchoring of the probe precedes the overall molecular rotation that, ultimately, controls the release.

As a final issue before analyzing the diffusive dynamics in these systems, we would like to comment on the possibility of investigating the protonated form of ibuprofen, IBUH. The choice of the anionic form, IBU, is granted to be adequate in the high $\mathrm{pH}$ regime, i.e., the UPS. However, at intermediate and particulary low $\mathrm{pH}$ values, it is not unlikely that the protonated form of ibuprofen might be present in major percentages within the nanochannel. Therefore, for the FPS system, we run MD trajectories in order to obtain the free energy profile for the IBUH release and compared them to those obtained for IBU. In Figure 10, we also include the Gibbs free energy profile for the release of IBUH from the silica/polyamine nanochannel. The IBUH profile looks vertically inverted with respect to that of IBU within the same FPS system. Moreover, one finds two barriers, of 1.5 and $2.7 \mathrm{kcal} \mathrm{mol}^{-1}$, whose positions (at $\xi \sim 11$ and $23 \AA$ ) coincide with those of the minima found in the IBU free energy profile. Note that, for the neutral IBUH, the nearby symmetric regions at both sides of the pore rim have now turned into the most unfavorable solvation environments. The key differences found between the free energy profiles of IBU and IBUH in the acid environment can be ascribed to the different net charge of the solute which, in the protonated IBUH case, becomes more stabilized at the center of the pore and at the low charge-density interfacial region of the pore outlet.

3.3. Diffusion. In this section, the translational mobility of water and ibuprofen species will be analyzed. To that aim, we computed the mean square displacements (MSDs) of the type

$$
\mathcal{R}_{\alpha}^{2}(t)=\left\langle\left|\mathbf{X}_{i}^{\alpha}(t)-\mathbf{X}_{i}^{\alpha}(0)\right|^{2}\right\rangle
$$

where $\left[\mathbf{X}_{i}^{\alpha}(t)-\mathbf{X}_{i}^{\alpha}(0)\right]$ denotes the projection of the displacement of the center-of-mass position of the $i$ th molecule of species $\alpha$ in $d$ dimensions. In the context of our nanochannel systems, the MSD time correlation of eq 9 has been computed considering all molecules whose center of mass lies, at $t=0$, within the $-15 \AA \leq z \leq 15 \AA$ region of the silica pore. From the Einstein equation,

$$
\lim _{t \rightarrow \infty} \frac{1}{2 d t} \mathcal{R}_{\alpha}^{2}(t)=D_{\alpha}
$$


(a)

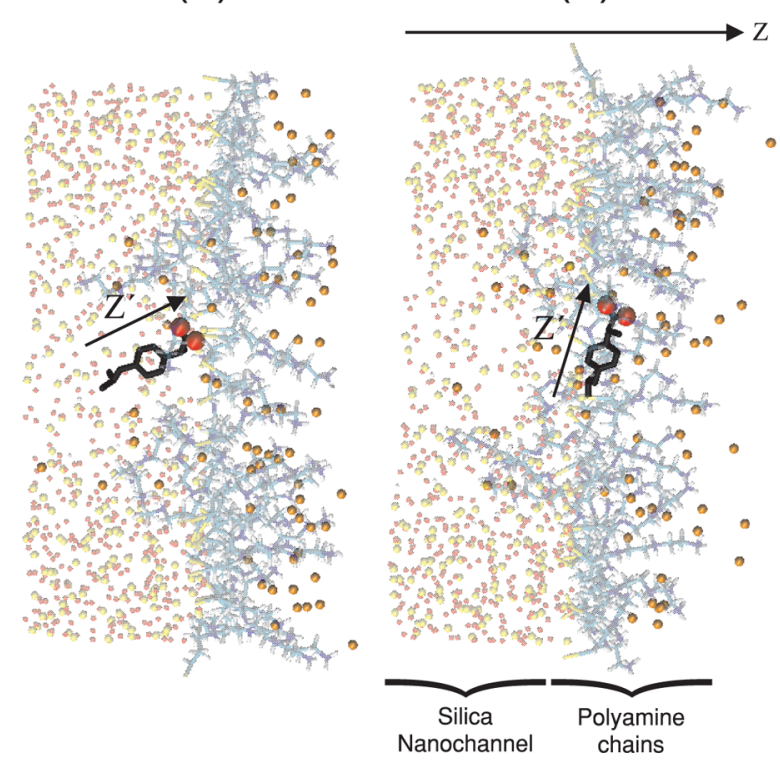

(c)

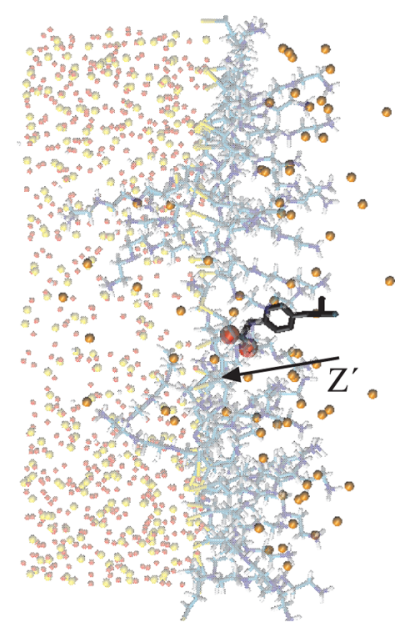

Figure 13. Representative snapshots during the three-step ibuprofen release from the silica nanochannel decorated with polyamine chains at the pore outlets. The $z^{\prime}$ axis, fixed to the ibuprofen molecular frame, shows the different orientations during the release mechanism.

the self-diffusion coefficient of species $\alpha, D_{\alpha}$, can be obtained. In the above equation, $d$ represents the dimensionality. Figure 14a shows the MSD of water species inside the nanochannel
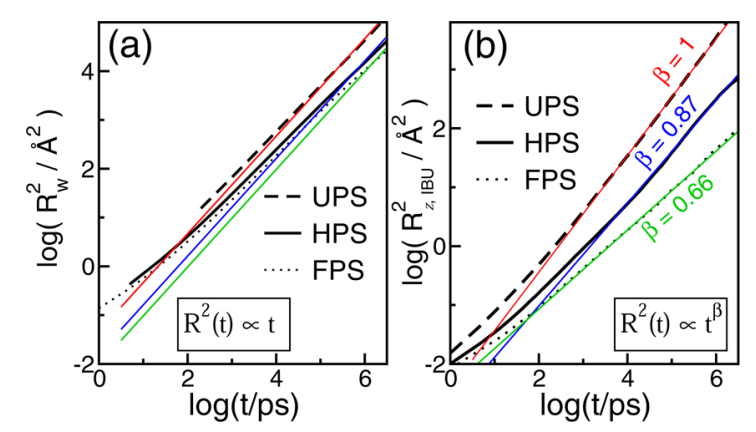

Figure 14. Mean square displacements of (a) water and (b) ibuprofen species, plotted in a log-log scale. The colored lines correspond to the fitting functions.

versus time, in a log-log scale. Although the mobility of water confined within the silica pore is smaller compared to that in the bulk phase, the plot shows that the MSD approaches the asymptote of slope unity after the initial transient. The diffusion coefficients obtained for water are listed in Table 2. Also listed are results for survival times, $\tau_{\mathrm{s}}$. These time scales correspond to the average temporal interval during which a given water molecule remains within the silica nanochannel and were

Table 2. Normalized Diffusion Coefficients for Water Species Trapped within the Silica Pore $\left(\tilde{D}_{\mathrm{w}}=D_{\mathrm{w}} / D_{\mathrm{w}}^{\text {bulk }}\right.$; with $\left.D_{\mathrm{w}}^{\text {bulk }}=2.4 \times 10^{-5} \mathrm{~cm}^{2} \mathrm{~s}^{-1}\right)$ and Survival Time, $\tau_{\mathrm{s}}$, Computed from the Survival Function

$\begin{array}{cccccc}\text { system } & \tilde{D}_{\mathrm{w}, x} & \tilde{D}_{\mathrm{w}, y} & \tilde{D}_{\mathrm{w}, z} & \tilde{D}_{\mathrm{w}} & \tau_{\mathrm{s}}(\mathrm{ns}) \\ \text { UPS } & 0.37 & 0.38 & 0.68 & 0.47 & 2.6 \\ \text { HPS } & 0.23 & 0.21 & 0.43 & 0.30 & 4.6 \\ \text { FPS } & 0.17 & 0.19 & 0.32 & 0.22 & 6.6\end{array}$

estimated from the decay of the survival time correlation function $^{56-58}$

$$
s(t)=\sum_{i}\left\langle p_{i}(0) p_{i}(t)\right\rangle
$$

where the characteristic function $p_{i}(t)$ equals 1 if molecule $i$ is found within the interior of the silica pore at time $t$ and zero otherwise. The values obtained for $\tau_{\mathrm{s}}$ depend, of course, on the nanochannel length; nevertheless, the fact that they are of the order of 2-6 ns guarantees that the diffusive regime of water molecules inside the pore is attained well before they escape toward the bulk reservoirs (note that the asymptotic behavior in the MSDs of Figure 14 is observed after $~ 300-400$ ps).

The results show that, in all cases, the overall normalized aqueous diffusion coefficients $\tilde{D}$ are smaller than that in bulk water. The translational mobility of water trapped within the pore slows down with the degree of polyamine protonationthat is, with the reduction of the pore aperture at the outletsbeing the largest retardation, the downshift found in the FPS system. Moreover, diffusive motions are highly anisotropic, ${ }^{43}$ with the axial diffusivity $\left(\tilde{D}_{\mathrm{w}, z}\right)$ being $\sim 82-88 \%$ larger than those computed along the radial directions $\left(\tilde{D}_{\mathrm{w}, x}, \tilde{D}_{\mathrm{w}, y}\right)$.

The translational mobility of the solute along the $z$-direction exhibits different and interesting features. Figure $14 \mathrm{~b}$ reveals that, except for UPS, the translational dynamics of IBU is subdiffusive, as described by a power law $\mathcal{R}^{2}(t) \propto t^{\beta}$, with $\beta \approx$ $0.66-0.87$. The deviation of $\beta$ with respect to unity increases with the acidity of the environment: at lower values of $\mathrm{pH}$, the anomalous character of the solute diffusion is enhanced. At high $\mathrm{pH}$ 's, the gates of the nanochannel are in an open state, and the polyamine chains do not bear any net charge; in this case, the polyamines behave like loose chains that allow diffusive mass transport in and out from the cavity, and do not exert any relevant Coulombic attraction with the IBU solute. At intermediate and low $\mathrm{pH}$ 's, the reduction of the pore aperture, along with the additional rigidity imposed by the Coulombic interactions between the protonated polyamine chains and 
chloride anions would promote an anomalous diffusion of ibuprofen along the axial direction.

The experimental results obtained by Casasús et al. ${ }^{33}$ also show distinct behaviors of the solute delivery kinetics in systems at intermediate and low pH's. More specifically, the authors show that the amount of solute release $(Q)$ in a high$\mathrm{pH}$ solution could be well described by a simple equation based on Fickian diffusion, through the Higuchi model, ${ }^{59,60}$ by $Q \propto$ $k(t)^{1 / 2}$. However, this latter functionality was not observed at intermediate and low pH's, where an exponential function of the type $Q \propto\left[\exp \left(k^{\prime} t\right)-1\right]$ was found to be more appropriate, in agreement with a first-order release kinetics. In contrast, Cosentino et al. ${ }^{61}$ reported that the diffusion profiles deviate substantially from those predicted by Fick's law. On the basis of a series of hypotheses, that differ from those considered in Higuchi's model, the authors propose an exponential dependence of the type $Q \propto\left[1-\exp \left(k^{\prime \prime} t\right)\right]$. From our perspective, it is not possible to gauge which is the most accurate temporal dependence of $Q$ vs $t$. Nevertheless, our analysis of the behavior of the ibuprofen mean square displacement with time does show deviations from the linear diffusive regime, revealing a non-Brownian, i.e., non-Fickean, dynamics for the release diffusion process. It is important to note that these observations agree not only with the experimental works mentioned above but also with many others reported in the literature. ${ }^{62-64}$

\section{CONCLUSIONS}

The molecular dynamics simulation results presented in this work provide new insights about microscopic structural details and dynamical information on the ibuprofen release from $\mathrm{pH}$ gated silica nanochannels. Inspired by experimental results, a $\mathrm{pH}$-driven gate-like ensemble was built by anchoring polyamine chains at the pore outlets of a cylindrical silica pore of diameter $\sim 30 \AA$ A. Effects from the $\mathrm{pH}$ of the solution were incorporated into the model by modifying the degree of polyamine protonation. High and low $\mathrm{pH}$ cases were investigated, corresponding to nonprotonated and fully protonated polyamine chains, respectively. An intermediate regime was also studied, by considering a suitable combination of non-, mono-, di-, and triprotonated polyamines. The increment of the acidity in the environment leads to an "open $\rightarrow$ close" mechanism of the gates, which is controlled by Coulomb repulsive interactions among ammonium groups of the polyamines, leading to a $72 \%$ reduction in the effective gate diamater, compared to what is found for the unprotonated system. It is worth mentioning that the quantitative reduction of the effective gate diameter should also be dependent on the size and net charge of the counteranion. Nevertheless, we believe that the qualitative behavior should be valid, regardless of the specific identity of the anion.

Using an ABF scheme, free energy profiles associated with a simple geometrical order parameter that describes the ibuprofen release from the nanochannel were constructed. The presence of a small barrier at the center of the pore in the IBU release profiles indicates that the central part of the pore cavity represents a highly unfavorable environment for IBU trapping. The profile in the UPS system exhibits a uniform 1 $\mathrm{kcal} \mathrm{mol}^{-1}$ well that extends from the interior of the channel up to the water bulk-like region outside the pore, with a small $\sim 0.8$ $\mathrm{kcal} \mathrm{mol}{ }^{-1}$ barrier, located $27 \AA$ away from the center of the pore, that arises from a subtle balance between the opposite trends found for water-water and solute-water contributions to the total free energy upon ibuprofen release.
The profiles computed at intermediate and low pH's exhibit an additional barrier centered at the pore rim, flanked by two symmetric local minima. More specifically, the microscopic analysis of the time evolution of a few relevant parameters allowed us to identify a three-step delivery mechanism of ibuprofen from $\mathrm{pH}$-gated nanochannels: During the first step, the IBU gets stacked inside the pore, near the outlet, with its hydrophilic carboxylate group pointing outward; the second step consists of a sudden molecular rotation around the stacking point; finally, in the third step, the hydrophilic moiety of IBU turns toward the interior of the pore and the solute is released to the aqueous bulk solution.

The free energy profile of a protonated form of ibuprofen, IBUH, was also computed for the system of highest acidity. The profile was found to be vertically inverted with respect to that of IBU in the same environment: we found two barriers, whose positions overlap with those of the two minima in the IBU profile. As such, due to the different net charge carried by the solute, the nearby symmetric regions, next to the gate, turned into the most unfavorable solvation environment.

The analysis of the translational dynamics of the solute in the axial direction showed anomalous diffusion features. At the highest acidity, a nonlinear power-law behavior was found for the temporal dependence of the mean square displacement. A subdiffusive dynamics was found for the delivery of IBU from the nanochannels, indicating a non-Brownian kinetics. Combined effects derived from the presence of a physical obstacle provided by the "closed" state of the gates, in addition to the rigidity imposed by the Coulombic interactions between protonated polyamine chains and chloride anions, would be responsible for a hindered and slower solute diffusion.

\section{AUTHOR INFORMATION}

\section{Corresponding Author}

*E-mail: javier@speedy.cnea.gov.ar.

\section{Notes}

The authors declare no competing financial interest.

\section{ACKNOWLEDGMENTS}

J.R. and M.D.E. thank CONICET for financial support (Grant PIP112-2008-00403). J.R. and M.D.E. are staff members of CONICET, Argentina.

\section{REFERENCES}

(1) Stupp, S. I.; Palmer, L. C. Supramolecular Chemistry and SelfAssembly in Organic Materials Design. Chem. Mater. 2014, 26, 507518

(2) Descalzo, A. B.; Martínez-Mañez, R.; Sancenón, F.; Hoffmann, K.; Rurack, K. The Supramolecular Chemistry of Organic-Inorganic Hybrid Materials. Angew. Chem., Int. Ed. 2006, 45, 5924-5948.

(3) Wang, H.; Yang, H.; Liu, H.; Yu, Y.; Xin, H. A Mesoporous Silica Nanocomposite Shuttle: pH-Triggered Phase Transfer Between Oil and Water. Langmuir 2013, 29, 6687-6696.

(4) Aznar, E.; Sancenón, F.; Marcos, M. D.; Martínez-Máñez, R.; Stroeve, P.; Cano, J.; Amorós, P. Delivery Modulation in Silica Mesoporous Supports via Alkyl Chain Pore Outlet Decoration. Langmuir 2012, 28, 2986-2996.

(5) Zimmerman, R; Basabe-Desmonts, L.; van der Baan, F.; Reinhoudt, D. A.; Crego-Calama, M. A Combinatorial Approach to Surface-Confined Cation Sensors in Water. J. Mater. Chem. 2005, 15, $2772-2777$

(6) Basabe-Desmonts, L.; Beld, J.; Zimmerman, R. S.; Hernando, J.; Mela, P.; García Parajó, M. F.; van Hulst, N. F.; van den Berg, A.; Reinhoudt, D. N.; Crego-Calama, M. A Simple Approach to Sensor 
Discovery and Fabrication on Self-Assembled Monolayers on Glass. J. Am. Chem. Soc. 2004, 126, 7293-7299.

(7) Montalti, M.; Prodi, L.; Zaccheroni, N.; Falini, G. SolventInduced Modulation of Collective Photophysical Processes in Fluorescent Silica Nanoparticles. J. Am. Chem. Soc. 2002, 124, 13540-13546.

(8) Montalti, M.; Prodi, L.; Zaccheroni, N. Fluorescence Quenching Amplification in Silica Nanosensors for Metal Ions. J. Mater. Chem. 2005, 15, 2810-2814.

(9) Chung, E.; Bizzotto, D.; Wolf, M. O. Potential-Controlled Switching of 2-(2'-Thienyl)Pyridine Adsorbed on $\mathrm{Au}(111)$. Chem. Commun. 2002, 3026-3027.

(10) Rampazzo, E.; Brasola, E.; Marcuz, S.; Mancin, F.; Tecila, P.; Tonellato, U. Surface Modification of Silica Nanoparticles: A New Strategy for the Realization of Self-Organized Fluorescence Chemosensors. J. Mater. Chem. 2005, 15, 2687-2696.

(11) Cooke, G. Electrochemical and Photochemical Control of HostGuest Complexation at Surfaces. Angew. Chem., Int. Ed. 2003, 42, $4860-4870$

(12) Corbellini, F.; Mulder, A.; Sartori, A.; Ludden, M. J. W.; Casnatti, A.; Ungaro, R.; Huskens, J.; Crego-Calama, M.; Reinhoudt, D. N. Assembly of a Supramolecular Capsule on a Molecular Printboard. J. Am. Chem. Soc. 2004, 126, 17050-17058.

(13) Wanunu, M.; Popovitz-Biro, R; Cohen, H.; Vaskevich, A.; Rubinstein, I. Coordination-Based Gold Nanoparticle Layers. J. Am. Chem. Soc. 2005, 127, 9207-9215.

(14) Banerjee, I. A.; Yu, L.; Matsui, H. Application of Host-Guest Chemistry in Nanotube Device Fabrication: Photochemically Controlled Immobilization of Azobenzene Nanotubes on Patterned $\alpha$-CD Monolayer/Au Substrates via Molecular Recognition. J. Am. Chem. Soc. 2003, 125, 9542-9543.

(15) Chen, Y.-f.; Banerjee, I. A.; Yu, L.; Djalali, R.; Matsui, H. Attachment of Ferrocene Nanotubes on $\beta$-Cyclodextrin SelfAssembled Monolayers with Molecular Recognitions. Langmuir 2004, 20, 8409-8413.

(16) Kay, E. R.; Leigh, D. A.; Zerbetto, F. Synthetic Molecular Motors and Mechanical Machines. Angew. Chem., Int. Ed. 2007, 46, $72-191$.

(17) Hernández, J. V.; Kay, E. R.; Leigh, D. A. A Reversible Synthetic Rotary Molecular Motor. Science 2004, 306, 1532-1537.

(18) Dietrich-Buchecker, C.; Jimenez-Molero, M. C.; V. Sartor, V.; Sauvage, J.-P. Rotaxanes and Catenanes as Prototypes of Molecular Machines and Motors. Pure Appl. Chem. 2003, 75, 1383-1393.

(19) Barboiu, M.; Lehn, J.-M. Dynamic Chemical Devices: Modulation of Contraction/Extension Molecular Motion by Coupled-Ion Binding/ $\mathrm{pH}$ Change-Induced Structural Switching. Proc. Natl. Acad. Sci. U. S. A. 2002, 75, 5201-5206.

(20) Balzani, V.; Credi, A.; Raymo, F. M.; Stoddart, J. F. Artificial Molecular Machines. Angew. Chem., Int. Ed. 2000, 39, 3348-3391.

(21) Vallet-Regí, M.; Bala, F.; Arcos, D. Mesoporous Materials for Drug Delivery. Angew. Chem., Int. Ed. 2007, 46, 7548-7558.

(22) Slowing, I. I.; Vivero-Escoto, J. L.; Wu, C.-W.; Lin, V. S.-Y. Mesoporous Silica Nanoparticles as Controlled Release Drug Delivery and Gene Transfection Carriers. Adv. Drug Delivery Rev. 2008, 60, $1278-1288$.

(23) He, Q.; Shi, J. Mesoporous Silica Nanoparticle Based Nano Drug Delivery Systems: Synthesis, Controlled Drug Release and Delivery, Pharmacokinetics and Biocompatibility. J. Mater. Chem. 2011, 21, 5845-5855.

(24) Mamaeva, V.; Rosenholm, J. M.; Bate-Eya, L. T.; Bergman, L.; Peuhu, E.; Duchanoy, A.; Fortelius, L. E.; Landor, S.; Toivola, D. M.; Lindén, M.; et al. Mesoporous Silica Nanoparticles as Drug Delivery Systems for Targeted Inhibition of Notch Signaling in Cancer. Mol. Ther. 2011, 19, 1538-1546.

(25) Argyo, C.; Weiss, v.; Bräuchle, C.; Bein, T. Multifunctional Mesoporous Silica Nanoparticles as a Universal Platform for Drug Delivery. Chem. Mater. 2014, 26, 435-451.
(26) Kresge, C. T.; Leonowicz, M. E.; Roth, W. J.; Beck, J. S. Ordered Mesoporous Molecular Sieves Synthesized by a Liquid-Crystal Template Mechanism. Nature (London) 1992, 359, 710-712.

(27) Zhao, D.; Feng, J.; Hup, Q.; Melosh, N.; Fredrickson, G. H.; Chmelka, B. F.; Stucky, G. D. Triblock Copolymer Syntheses of Mesoporous Silica with Periodic 50 to 300 Angstroms Pores. Science 1998, 279, 548-552.

(28) Liu, Y.; Zhang, W.; Pinnavaia, T. J. Steam-Stable MSU-S Aluminosilicate Mesostructures Assembled from Zeolite ZSM-5 and Zeolite Beta Seeds. Angew. Chem., Int. Ed. 2001, 40, 1255-1258.

(29) Han, Y.; Li, D.; Zhao, L.; Song, J.; Yang, X.; Li, N.; Di, Y.; Li, C.; $\mathrm{Wu}, \mathrm{S}$.; $\mathrm{Xu}, \mathrm{X}$; et al. High-Temperature Generalized Synthesis of Stable Ordered Mesoporous Silica-Based Materials by Using Fluorocarbon-Hydrocarbon Surfactant Mixtures. Angew. Chem., Int. Ed. 2003, 42, 3633-3637.

(30) Beck, J. S.; Vartuli, J. C.; Roth, W. J.; Leonowicz, M. E.; Kresge, C. T.; Schmitt, K. D.; Chu, C. T. W.; Olson, D. H.; Sheppard, E. W. A New Family of Mesoporous Molecular Sieves Prepared with Liquid Crystal Templates. J. Am. Chem. Soc. 1992, 114, 10834-10843.

(31) Wight, A. P.; Davis, M. E. Design and Preparation of OrganicInorganic Hybrid Catalysts. Chem. Rev. 2002, 102, 3589-3614.

(32) Casasús, R.; Marcos, M. D.; Martínez-Mañez, R.; Ros-Lis, J. V.; Soto, J.; Villaescusa, L. A.; Amorós, P.; Beltrán, D.; Guillem, C.; Latorre, J. Toward the Development of Ionically Controlled Nanoscopic Molecular Gates. J. Am. Chem. Soc. 2004, 126, 86128613.

(33) Casasús, R.; Climent, E.; Marcos, M. D.; Martínez-Máñez, R.; Sancenón, F.; Soto, J.; Amorós, P.; Cano, J.; Ruiz, E. Dual Aperture Control on $\mathrm{pH}$ - and Anion-Driven Supramolecular Nanoscopic Hybrid Gate-Like Ensembles. J. Am. Chem. Soc. 2008, 130, 19031917.

(34) Gao, Q.; Xu, Y.; Wu, D.; Sun, Y.; Li, X. pH-Responsive Drug Release from Polymer-Coated Mesoporous Silica Spheres. J. Phys. Chem. C 2009, 113, 12753-12758.

(35) Gao, Q.; Xu, Y.; Wu, D.; Shen, W.; Deng, F. Synthesis, Characterization, and In Vitro $\mathrm{pH}$-Controllable Drug Release from Mesoporous Silica Spheres with Switchable Gates. Langmuir 2010, 26, 17133-17138.

(36) Gao, Q.; Chen, Z.; Xu, J.; Xu, Y. pH-Controlled Drug Release from Mesoporous Silica Spheres with Switchable Gates. Adv. Mater. Res. 2011, 236-238, 2142-2145.

(37) Berendsen, H. J. C.; Grigera, J. R.; Straatsma, T. P. The Missing Term in Effective Pair Potentials. J. Phys. Chem. 1987, 91, 6269-6271.

(38) Rodriguez, J.; Elola, M. D.; Laria, D. Confined Polar Mixtures Within Cylindrical Nanocavities. J. Phys. Chem. B 2010, 114, 79007908.

(39) MacKerell, J. A. D.; Banavali, N.; Foloppe, N. Development and Current Status of the CHARMM Force Field for Nucleic Acids. Biopolymers 2001, 56, 257-265.

(40) Neria, E.; Fischer, S.; Karplus, M. Simulation of Activation Energies in Molecular Systems. J. Chem. Phys. 1996, 105, 1902-1921.

(41) Raman, E. P.; Takeda, T.; Klimov, D. K. Molecular Dynamics Simulations of Ibuprofen Binding to A $\beta$ Peptides. Biophys. J. 2009, 97, 2070-2079.

(42) Chang, W. E.; Takeda, T.; Raman, E. P.; Klimov, D. K. Molecular Dynamics Simulations of Anti-Aggregation Effect of Ibuprofen. Biophys. J. 2010, 98, 2662-2670.

(43) Elola, M. D.; Rodriguez, J.; Laria, D. Structure and Dynamics of Liquid Methanol Confined Within Functionalized Silica Nanopores. J. Chem. Phys. 2010, 133, 154707-154715.

(44) Elola, M. D.; Rodriguez, J.; Laria, D. Liquid Methanol Confined Within Functionalized Silica Nanopores. II: Solvation Dynamics of Coumarin 153. J. Phys. Chem. B 2011, 115, 12859-12867.

(45) Kamijo, T.; Yamaguchi, A.; Suzuki, S.; Teramae, N.; Itoh, T.; Ikeda, T. Solvation Dynamics of Coumarin 153 in Alcohols Confined in Silica Nanochannels. J. Phys. Chem. A 2008, 112, 11535-11542.

(46) Strömgaard, K.; Piazzi, L.; Olsen, C. A.; Franzyk, H.; Jaroszewski, J. W. Protolytic Properties of Polyamine Wasp Toxin 
Analogues Studied by 13C NMR Spectroscopy. Magn. Reson. Chem.

2006, 44, 1013-1022.

(47) Stumm, W. Aquatic Surface Chemistry: Chemical Processes at the Particle-Water Interface; Interscience Publication, John Wiley \& Sons, Inc.: New York, 1987; Chapter 4.

(48) Hague, D. N.; Moreton, A. D. Protonation Sequence of Linear Aliphatic Polyamines by 13C NMR Spectroscopy. J. Chem. Soc., Perkin Trans. 2 1994, 2, 265-270.

(49) Phillips, J. C.; Braun, R.; Wang, W.; Gumbart, J.; Tajkhorshid, E.; Villa, E.; Chipot, C.; Skeel, R. D.; Kale, L.; Schulten, K. Scalable MD with namd. J. Comput. Chem. 2005, 26, 1781-1802.

(50) Tolpekina, T. V.; den Otter, W. K.; Briels, W. J. Simulations of Stable Pores in Membranes: System Size Dependence and Line Tension. J. Chem. Phys. 2004, 121, 8014-8020.

(51) Darve, E.; Pohorille, A. Calculating Free Energies Using Average Force. J. Chem. Phys. 2001, 115, 9169-9183.

(52) Rodriguez-Gomez, D.; Darve, E.; Pohorille, A. Assessing the Efficiency of Free Energy Calculation Methods. J. Chem. Phys. 2004, 120, 3563-3578.

(53) Rodriguez, J.; Elola, M. D. Encapsulation of Small Ionic Molecules Within $\alpha$-Cyclodextrins. J. Phys. Chem. B 2009, 113, 14231428.

(54) Yu, Y.; Chipot, C.; Cai, W.; Shao, X. Molecular Dynamics Study of the Inclusion of Cholesterol Into Cyclodextrins. J. Phys. Chem. B 2006, 110, 6372-6378.

(55) Yu, Y.; Chipot, C.; Sun, T.; Shao, X. Spatial Arrangement of $\alpha$ Cyclodextrins in a Rotaxane. Insights from Free-Energy Calculations. J. Phys. Chem. B 2008, 112, 5268-5271.

(56) Rocchi, C.; Bizzarri, A. R.; Cannistraro, S. Water Dynamical Anomalies Evidenced by Molecular Dynamics Simulations at the Solvent-Protein Interface. Phys. Rev. E 1998, 57, 3315-3325.

(57) Impey, R. W.; Madden, P. A.; McDonald, I. R. Hydration and Mobility of Ions in Solution. J. Phys. Chem. 1983, 87, 5071-5083.

(58) Martins, L. R; Ribeiro, M. C. C.; Skaf, M. S. Molecular Dynamics Simulations of the Squarate Dianion in Aqueous Solution. J. Phys. Chem. B 2002, 106, 5492-5499.

(59) Higuchi, T. Rate of Release of Medicaments from Ointment Bases Containing Drugs in Suspension. J. Pharm. Sci. 1961, 50, 874875.

(60) Higuchi, T. Mechanism of Sustained-Action Medication. Theoretical Analysis of Rate of Release of Solid Drugs Dispersed in Solid Matrices. J. Pharm. Sci. 1963, 52, 1145-1149.

(61) Cosentino, C.; Amato, F.; Walczak, R.; Boiarski, A.; Ferrari, M. Dynamic Model of Biomolecular Diffusion Through Two-Dimensional Nanochannels. J. Phys. Chem. B 2005, 109, 7358-7364.

(62) Kukla, V.; Kornatowski, J.; Demuth, D.; Girnus, I.; Pfeifer, H.; Rees, L. V. C.; Schunk, S.; Unger, K. K.; Kärger, J. NMR Studies of Single-File Diffusion in Unidimensional Channel Zeolites. Science 1996, 272, 702-704.

(63) Wei, Q. H.; Bechinger, C.; Leiderer, P. Single-File Diffusion of Colloids in One-Dimensional Channels. Science 2000, 287, 625-627.

(64) Lin, B.; Yu, J.; Rice, S. A. Direct Measurements of Constrained Brownian Motion of an Isolated Sphere Between Two Walls. Phys. Rev. E 2000, 62, 3909-3919. 